\title{
Cytochrome P450 metabolic resistance (CYP6P9a) to pyrethroids imposes a fitness cost in the major African malaria vector Anopheles funestus
}

\author{
Magellan Tchouakui $i^{1,2} \cdot$ Jacob Riveron Miranda ${ }^{1,3} \cdot$ Leon M. J. Mugenzi ${ }^{1,4} \cdot$ Doumani Djonabaye ${ }^{1,5} \cdot$

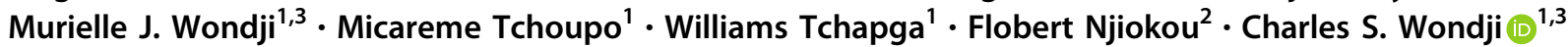

Received: 21 October 2019 / Revised: 26 February 2020 / Accepted: 27 February 2020

(c) The Author(s) 2020. This article is published with open access

\begin{abstract}
Metabolic resistance threatens the sustainability of pyrethroid-based malaria control interventions. Elucidating the fitness cost and potential reversal of metabolic resistance is crucial to design suitable resistance management strategies. Here, we deciphered the fitness cost associated with the CYP6P9a (P450-mediated metabolic resistance) in the major African malaria vector Anopheles funestus. Reciprocal crosses were performed between a pyrethroid susceptible (FANG) and resistant (FUMOZ-R) laboratory strains and the hybrid strains showed intermediate resistance. Genotyping the CYP6P9a-R resistance allele in oviposited females revealed that $C Y P 6 P 9 a$ negatively impacts the fecundity as homozygote susceptible mosquitoes (CYP6P9a-SS) lay more eggs than heterozygote $(\mathrm{OR}=2.04: P=0.01)$ and homozygote resistant mosquitoes. CYP6P9a also imposes a significant fitness cost on the larval development as homozygote resistant larvae (CYP6P9a-RR) developed significantly slower than heterozygote and homozygote susceptible mosquitoes $\left(\chi^{2}=11.2 ; P=0.0008\right)$. This fitness cost was further supported by the late pupation of homozygote resistant than susceptible mosquitoes $(\mathrm{OR}=2.50 ; P<0.01)$. However, СYP6P9a does not impact the longevity as no difference was observed in the life span of mosquitoes with different genotypes $\left(\chi^{2}=1.6 ; P=0.9\right)$. In this hybrid strain, a significant decrease of the resistant CYP6P9a-RR genotype was observed after ten generations $\left(\chi^{2}=6.6 ; P=0.01\right)$ suggesting a reversal of P450-based resistance in the absence of selection. This study shows that the $\mathrm{P} 450$-mediated metabolic resistance imposes a high fitness cost in malaria vectors supporting that a resistance management strategy based on rotation could help mitigate the impact of such resistance.
\end{abstract}

Associate editor: Gerald Heckel

The original version of this article was revised due to a retrospective Open Access order.

Supplementary information The online version of this article (https:// doi.org/10.1038/s41437-020-0304-1) contains supplementary material, which is available to authorised users.

Magellan Tchouakui

magellan.tchouakui@crid-cam.net

$\triangle$ Charles S. Wondji

charles.wondji@1stmed.ac.uk

1 Centre for Research in Infectious Diseases (CRID), P.O. Box 13501, Yaoundé, Cameroon

2 Parasitology and Ecology Laboratory, Department of Animal Biology and Physiology, Faculty of Science, University of Yaoundé 1, P.O. Box 812, Yaoundé, Cameroon

\section{Background}

Malaria control relies mainly on insecticide-based interventions, notably pyrethroid-based long-lasting insecticidal nets (LLINs) and indoor residual spraying (IRS). Significant efforts have been made globally to eliminate malaria leading to consistent reduction in malaria cases and mortality in Africa by $42 \%$ and $66 \%$, respectively (Bhatt et al. 2015; Riveron et al. 2018). However, increasing insecticide

3 Department of Vector Biology, Liverpool School of Tropical Medicine, Pembroke Place, L35QA Liverpool, UK

4 Department of Biochemistry and Molecular Biology, Faculty of Science, University of Buea, P.O. Box 63, Buea, Cameroon

5 Pharmacology and Toxicology Laboratory, Department of Biochemistry, Faculty of Science, University of Yaoundé 1, P.O. Box 812, Yaoundé, Cameroon 
resistance in malaria vector species presents a major challenge to these vector control interventions and likely contributed to the increase in malaria incidence in the last 2 years (WHO 2018). To sustain the effectiveness of these interventions it is imperative to implement suitable insecticide resistance management (IRM) strategies to reduce the negative impact of such resistance. IRM strategies such as rotation of insecticide classes, rely on resistance alleles having a fitness cost inducing a selection against resistance alleles in the absence of insecticide selection pressure. Therefore, understanding the fitness cost that selection can act against mosquitoes is a key pre-requisite to effective IRM. Since pyrethroids are by far the most widely used insecticide class and the main one recommended for the impregnation of bed nets, elucidating the fitness costs of molecular mechanisms conferring pyrethroid resistance in mosquitoes could guide suitable control measures for malaria prevention.

The two main mechanisms driving pyrethroid resistance are target-site resistance (e.g., knockdown resistance, $k d r$ ) and metabolic resistance through over-expression of detoxification enzymes (e.g., cytochrome P450s, glutathione S-transferases and esterases) (Ranson et al. 2011; Riveron et al. 2018). Target-site resistance through $k d r$ is well characterised, and the DNA-based diagnostic tools, were designed in the late 1990s (Martinez-Torres et al. 1998; Ranson et al. 2000). This allowed studying the fitness cost of target-site resistance on different life traits in a range of mosquito species including their reproduction, developmental time of immature stages, adult longevity and vector competence (Alout et al. 2016; Alout et al. 2014; Assogba et al. 2015; Brito et al. 2013; Martins et al. 2012). These studies highlighted that resistant vectors may have lower mating success, lower fecundity and fertility, higher developmental time and reduced longevity. The presence of such fitness costs that can impact the spread and persistence of resistance alleles in the vector populations is a prerequisite for the implementation of most IRM strategies including rotation of insecticides. In contrast, metabolic resistance, a very common resistance mechanism in mosquitoes and considered to be more likely to cause control failure (Hemingway 2014), still had no molecular diagnostic tools, despite progress made in elucidating its molecular basis (Edi et al. 2014; Ibrahim et al. 2015; Mitchell et al. 2012; Riveron et al. 2013). This has prevented assessment of the fitness cost associated with this resistance mechanism and consequently hampered the design of suitable resistance management strategy to control malaria vectors. However, recent progress has been made in detecting key markers of metabolic resistance in major malaria vectors including for glutathione S-transferase mediated resistance such as the L119F-GSTe2 marker in Anopheles funestus (Riveron et al. 2014) and the I114T-
GSTe2 in An. gambiae (Mitchell et al. 2012). The design of the L119F-GSTe2 diagnostic tool recently allowed assessment of the fitness cost of GST-based metabolic resistance revealing significant cost in the GST-resistant mosquitoes, although also revealing that they live longer (Tchouakui et al. 2018) and are more infected with Plasmodium (Tchouakui et al. 2019). Recently, major progress was also made in detecting molecular marker for cytochrome P450based resistance with the detection of $c i s$-regulatory variants driving the expression of the major pyrethroid resistance gene CYP6P9a in An. funestus (Weedall et al. 2019). The simple PCR-RFLP assay designed has already helped to demonstrate that pyrethroid resistance is reducing the efficacy of LLINs as resistant mosquitoes were shown to significantly survive exposure to these nets and also blood fed more than the susceptible ones (Weedall et al. 2019). This diagnostic tool also provides an opportunity to assess the fitness cost of P450-mediated metabolic resistance in malaria vectors. An. funestus is particularly a suitable vector for assessing the impact of metabolic resistance as this mechanism is the main cause of pyrethroid resistance with the absence of $k d r$ mutations consistently reported in populations of this malaria vector throughout Africa (Amenya et al. 2008; Okoye et al. 2008; Wondji et al. 2011; Riveron et al. 2013; Irving and Wondji 2017). One of the limitation of the studies on fitness cost is the use of resistant and susceptible strains from different geographical origins as they may differ in many other genes than those involved in resistance. For this reason, to minimise the effect of the genetic background on related fitness parameters, we proceeded by a crossing between the resistant and the susceptible strains so that the genetic background of the resistant strain is shared with that of the susceptible one as described elsewhere (Amin and White 1984; Argentine et al. 1989).

We elucidated the fitness cost of P450-based metabolic resistance on life traits of the malaria vector An. funestus, to better inform the design of suitable resistance management strategies against malaria vectors. This revealed a significant negative impact on fecundity and larval development of resistant mosquitoes CYP6P9a-RR while demonstrating a significant reduction of the frequency of resistant allele in the absence of selection.

\section{Methods}

\section{Establishment of the mosquito strains}

Reciprocal crosses were performed in January 2017 between FANG and FUMOZ-R, two An. funestus laboratory strains for several generations in order to bring the CYP6P9a resistance into a susceptible genetic background. 
FUMOZ-R is a pyrethroid (permethrin) resistant selected strain originates from southern Mozambique (FUMOZ-R) (Hunt et al. 2005) and kept in colony since July 2001. This strain was selected based on its resistance status after $1 \mathrm{~h}$ exposure to permethrin after WHO bioassays (WHO 1998) and currently exhibits $0 \%$ mortality at $0.75 \%$ permethrin exposure. The FANG strain originates from southern Angola and kept in colony since January 2003 and is fully susceptible to all major vector control insecticides. Previous studies have shown that the CYP6P9a alleles conferring resistance in southern Africa is fixed in the FUMOZ-R strain, whereas it is absent in FANG (Weedall et al. 2019). To perform the crossing, pupae of each strain were collected and put individually in $15 \mathrm{ml}$ falcon tubes for individual emergence then, the males of the resistant strain were mixed into a same cage with the females of the susceptible colony (and reciprocally) for random mating to generate the first generation.

\section{Mosquito's rearing}

In each generation, eggs obtained from the crosses between both strains were transferred in paper cups containing mineral water for hatching. These eggs were flushed each day with mineral water and 2-3 days post-hatching, larvae obtained were transferred in larvae bowl and reared in mineral water with Tetramin baby fish food everyday as described previously (Morgan et al. 2010). Water of each larvae bowl was changed every 2 days. The $F_{1}$ adults generated were randomly mixed in cages and fed with $10 \%$ sugar solution for crossing and production of the next generation. In each generation, after emergence of the adults, mosquitoes were let to randomly mate in cages for 5 days and blood fed three times before been allowed to lay eggs for the next generation. After the initial $F_{1}$ generation obtained from the reciprocal crosses, the hybrid strain FANG/FUMOZ-R (hybrid stain from female FANG and males FUMOZ-R) was reared till $\mathrm{F}_{10}$ generation in order to assess the fitness cost and a potential reversal of resistance.

\section{Susceptibility profile of the hybrid FANG/FUMOZ-R strain and validation of the implication of CYP6P9a in the resistance}

WHO bioassays were carried out to assess the susceptibility profile of the two reciprocal hybrid strains for pyrethroids ( $0.75 \%$ permethrin and $0.05 \%$ deltamethrin), DDT $(4 \%)$ and the carbamate bendiocarb $(0.1 \%)$. The bioassays were performed according to WHO protocol (WHO 2013). In order to investigate the correlation between the CYP6P9a marker and pyrethroid resistance, additional bioassays were conducted with permethrin and deltamethrin for $30 \mathrm{~min}$ and $90 \mathrm{~min}$. Alive mosquitoes after $90 \mathrm{~min}$ of exposure and those dead after $30 \mathrm{~min}$ of exposition were then genotyped to establish the association between the CYP6P9a-R resistant allele and the ability of mosquitoes to survive to these insecticides.

\section{Life traits experiments}

All parameters were evaluated by simultaneously comparing fitness parameters between homozygotes resistant (CYP6P9a-RR), heterozygotes (CYP6P9a-RS) and homozygote susceptible (CYP6P9a-SS), reared together in the same containers and under the same environmental conditions such as larval density and feeding, temperature and light cycle.

\section{Fecundity and fertility}

In the 4 th generation $\left(\mathrm{F}_{4}\right)$, after $4-5$ days of mating in cages, females were blood fed three times and given 4 days to become fully gravid. Fully gravid-females were put individually in $1.5 \mathrm{ml}$ Eppendorf tubes with damp filter paper to enable them to lay eggs as previously described (Morgan et al. 2010). The number of eggs laid per female and the number of larvae obtained after hatching were recorded. As a Shapiro-Wilk normality test showed non-normal distribution of eggs, the impact of resistance on fecundity was assessed by comparing the median number of eggs laid by different genotypes using a Kruskal-Wallis non-parametric test. In addition, odds ratio for oviposition between CYP6P9a-RR, CYP6P9a-SS and CYP6P9a-RS was also assessed using a statistical significance calculation based on the Fisher's exact probability test. The impact of resistance on fertility was assessed by comparing the hatch rate between the CYP6P9a genotypes using a Chi square test.

\section{Larval and pupal development}

After recording the total number of larvae produced per female, all larvae comprising the three CYP6P9a genotypes were pooled and reared together in the same larvae bowl, thus avoiding variation in environmental conditions. This experiment was performed in three replicates of ten trays per replicate and all immature stages were reared in the standard insectary condition. In order to prevent overcrowding and competition for food, larval bowls used were large enough to accommodate all larvae. The number of larvae varied between 200 and 300 per tray and water was changed every 2 days in each tray to minimise the effect of pollution from the food according to Morgan et al. (2010). Changes in the time of development of immature stages and mortality rates was equally assessed by genotyping about 100 larvae at different stages (L1, L2, L3 and L4). For this purpose, genotype frequency was monitored in each stage 
of development. The dynamic of pupae formation was evaluated by comparing the genotype and allele frequencies from the first day of pupation (pupae day 9), in the third day of pupation (pupae day 11) and in the fifth day of pupation (pupae day 13). Chi-squared and odds ratio with Fisher's exact test were used to assess significance of the difference in genotype distribution between larval stages and pupae obtained at different time-points.

\section{Adult longevity}

After emergence of adults, a set of about 150 mosquitoes was removed from the cages at different time-points (day 1, 10, 20 and 30 after emergence). On average, 100 mosquitoes were used for genotyping whereas three pools of ten mosquitoes each were used to assess the gene expression level of $C Y P 6 P 9 a$ at each time-point. The life span of homozygous resistant adult mosquitoes was compared with that of susceptible and heterozygotes (RS) mosquitoes by assessing the frequency of $C Y P 6 P 9 a$ genotypes/alleles and the expression level of $C Y P 6 P 9 a$ (qRT-PCR) at different time-points.

\section{Population cage experiments to assess a potential reversal to susceptibility}

The dynamics of CYP6P9a-R resistant allele frequency in the absence of insecticide pressure was assessed using cage experiments. After crossing between female FANG and male FUMOZ-R, the progeny obtained were let in cages for intercrosses for ten generations. In each generation, all mosquitoes irrespective of their genotypes were randomly mixed in cages for intercrossing to generate the next generation. Each generation consisted in about three cages of at least 200 mosquitoes/cage of mixed genotypes. In the first generation, the frequency of the $C Y P 6 P 9 a \_R$ resistant allele was assessed and then monitored in following generations by genotyping a set of about 75 females aged between 2 and 5 days old.

\section{Genotyping of the CYP6P9a resistance allele using PCR-RFLP}

Genomic DNA was extracted from adult mosquitoes and all larval and pupal stages using the Livak method (Livak 1984). The genotyping of CYP6P9a resistance allele was done using PCR-RFLP method as recently described (Weedall et al. 2019). The RFLP6P9aF forward primer, 5'TCC CGA AAT ACA GCC TTT CAG-3 and RFLP6P9aR 5'-ATT GGT GCC ATC GCT AGA AG-3' reverse primers are used in the first step amplification of the partial CYP6P9a upstream region. PCR reactions were carried out on genomic DNA from individual mosquitoes. The final
$15 \mu \mathrm{l}$ PCR mixture contained $1.5 \mu \mathrm{l}$ of 10X KAPA Taq buffer A (KAPA Biosystems), $0.12 \mu \mathrm{l}$ of $5 \mathrm{U} / \mu \mathrm{l}$ KAPA Taq polymerase, $0.12 \mu \mathrm{l}$ of $25 \mu \mathrm{M}$ dNTP, $0.75 \mu \mathrm{l}$ of $25 \mu \mathrm{M}$ $\mathrm{MgCl} 2,0.51 \mu \mathrm{l}$ of each primer, $10.49 \mu \mathrm{l}$ of $\mathrm{dH} 2 \mathrm{O}$, and $1 \mu \mathrm{l}$ of genomic DNA. The PCR parameters were $95^{\circ} \mathrm{C}$ for $5 \mathrm{~min}$ and 35 cycles of $94^{\circ} \mathrm{C}$ for $30 \mathrm{~s}, 58^{\circ} \mathrm{C}$ for $30 \mathrm{~s}$, and $72{ }^{\circ} \mathrm{C}$ for $45 \mathrm{~s}$, followed by a final extension step of $72{ }^{\circ} \mathrm{C}$ for $10 \mathrm{~min}$. The size of PCR products was obtained on $1.5 \%$ agarose gel stained with Midori Green Advance DNA Stain (Nippon genetics Europe $\mathrm{GmbH}$, Dueren, Germany) and visualised using a gel imaging system to confirm the product size $(450 \mathrm{bp})$. For the second step, the TaqI enzyme (restriction site $\left(5^{\prime}-\mathrm{TCGA}-3^{\prime}\right)$ ) was used to digest the PCR product and detect the CYP6P9a_R resistant allele as previously described (Weedall et al. 2019). For this second step, $10 \mu \mathrm{l}$ of the digestion mix is made using $1 \mu \mathrm{l}$ of CutSmart buffer, $0.2 \mu$ l of 2 units of TaqI restriction enzyme (New England Biolabs, Ipswich, MA, USA), $5 \mu$ of PCR product, and $3.8 \mu \mathrm{l}$ of $\mathrm{dH}_{2} \mathrm{O}$. The mix was incubated at $65^{\circ} \mathrm{C}$ for $2 \mathrm{~h}$ and the product separated on $2.0 \%$ agarose gel stained with Midori Green. After this second step, CYP6P9a-RR displays one band at $350 \mathrm{bp}$, the CYP6P9aSS showed one band as well at $450 \mathrm{bp}$ whereas heterozygotes individuals present both bands.

\section{Expression profile of CYP6P9a and adult longevity using qRT-PCR}

The quantitative reverse-transcription PCR (qRT-PCR) was performed to assess the expression level of $C Y P 6 P 9 a$ from day 1 to day 30. Total RNA from three biological replicates (ten mosquitoes each) from day 1, day 10, day 20 and day 30 after adult emergence was extracted using the Picopure RNA Isolation Kit (Arcturus). One micrograms of RNA from each of the three biological replicates at each timepoint, and FANG (full susceptible strain) was used as a template for cDNA synthesis using the superscript III (Invitrogen) with oligo-dT20 and RNase H, following the manufacturer's instructions. The qRT-PCR was carried out in a MX3005 real-time PCR system (Agilent) using Brilliant III Ultra-Fast SYBR Green qPCR Master Mix (Agilent). A total of $10 \mathrm{ng}$ of cDNA from each sample was used as template in a three-step programme involving a denaturation at $95^{\circ} \mathrm{C}$ for $3 \mathrm{~min}$ followed by 40 cycles of $10 \mathrm{~s}$ at $95^{\circ} \mathrm{C}$ and $10 \mathrm{~s}$ at $60^{\circ} \mathrm{C}$ and a last step of $1 \mathrm{~min}$ at $95^{\circ} \mathrm{C}$, $30 \mathrm{~s}$ at $55^{\circ} \mathrm{C}$, and $30 \mathrm{~s}$ at $95^{\circ} \mathrm{C}$ as previously described (Kwiatkowska et al. 2013; Riveron et al. 2013). The relative expression level and fold-change (FC) of CYP6P9a in each time-point relative to the susceptible strain was calculated according to the $2^{-\Delta \Delta C T}$ method (Schmittgen and Livak 2008) after normalisation with the housekeeping genes ribosomal protein S7 (RSP7; AFUN007153) and actin 5C (AFUN006819). 


\section{Results}

\section{Susceptibility profiles of the FUMOZ-R/FANG and FANG/FUMOZ-R strains}

Bioassays conducted on the $\mathrm{F}_{4}$ mosquitoes from the reciprocal crosses between females FANG/males FUMOZ-R (FANG/FUMOZ-R) and females FUMOZ-R/males FANG (FUMOZ-R/FANG) strains revealed that both hybrid strains were resistant to pyrethroids and carbamates as previously described by Weedall et al. (2019). Both strains were moderately resistant to DDT (93\% mortality). For deltamethrin, a higher mortality rate was recorded for the strain generated from the crossing between females FUMOZ_R and males FANG ( $48.5 \%$ mortality) compared with the strain obtained from females FANG and males FUMOZ_R (77.3\%) (Fig. S1). For bendiocarb (carbamate), the resistance pattern was similar in both reciprocal strains (Weedall et al. 2019).

\section{Validation of the role of the CYP6P9a metabolic resistance in the observed pyrethroid resistance}

Assessment of the differential expression of $C Y P 6 P 9 a$ in the hybrid strain after exposure to permethrin and deltamethrin revealed that the expression level of this gene was as followed: permethrin alive: $\mathrm{FC}=16.6 \pm 4.7, P=0.01$; deltamethrin alive: $\mathrm{FC}=8.8 \pm 4.7, P=0.04$ and unexposed mosquitoes $\mathrm{FC}=7.9 \pm 2.12, P=0.01$. For both groups, this level of expression was significantly high compared with the susceptible strain FANG supporting that $C Y P 6 P 9 a$ plays a role in the resistance observed (Fig. 1b). Furthermore, the level of expression did not differ significantly between exposed and unexposed mosquitoes (Fig. 1a) showing that the gene is constitutively expressed in this line. To validate the role of the recently discovered CYP6P9a_R resistant allele in the observed pyrethroid resistance we assessed the correlation between this allele and the ability of mosquitoes to survive after exposure. Mortality rates of $39.0 \%$ and $42.3 \%$ after 3 -min exposure and mortality of $81.3 \%$ and $86.3 \%$ after 90 -min exposure were obtained for permethrin and deltamethrin, respectively (Fig. 1c). Genotyping of the dead mosquitoes after 30-min exposure and the alive after 90-min exposure to these insecticides as previously described showed that the ability of homozygotes resistant mosquitoes to survive after exposition was higher compared with the homozygous susceptible (SS) as recently also described (Weedall et al. 2019).

\section{Influence of the CYP6P9a_R on the fecundity/ fertility of female mosquitoes}

No significant difference was observed in the distribution of genotypes between females which have successfully laid eggs after blood feeding and those which did not laid eggs $\left(x^{2}=4.3 ; \mathrm{df}=2 ; p>0.1\right)$. However, a higher but not significant $\left(x^{2}=1.65 ; p=0.19\right)$ frequency of CYP6P9a_R resistant allele was observed in non-oviposited females (39\%) compared with the oviposited females (33\%). Assessment of the odd ratio (OR) for oviposition between homozygote resistant (RR) mosquitoes, SS and RS mosquitoes using Fisher's exact probability test revealed that the ability of SS mosquitoes to lay eggs was higher compared with RS (OR $=2.04$; confidence interval (CI) 95\%: 1.1-3.8; $p=0.01)$. The same trend was observed when compared with RR although not significant $(\mathrm{OR}=$ 2.0; CI 95\%: 0.7-5.7; $p=0.15$ ) (Fig. 2a, b) suggesting that mosquitoes harbouring the resistant allele have less chance to lay eggs compared with those with the susceptible allele. RS mosquitoes displayed the same ability of oviposition than $\mathrm{RR}(\mathrm{OR}=1$; CI 95\%: $0.4-2.6 ; p=0.57)$
(A)

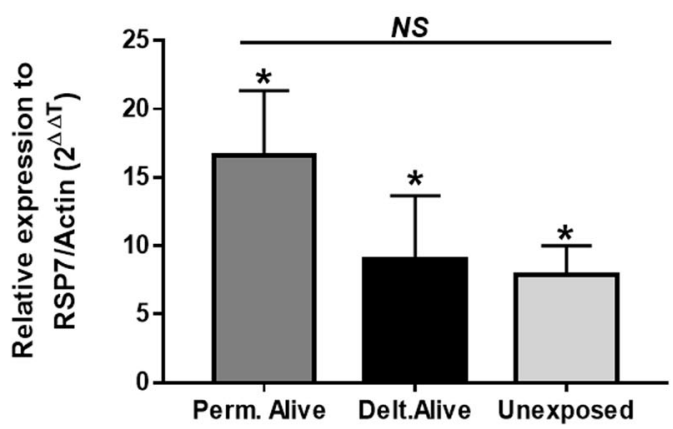

Fig. 1 Validation of the implication of CYPGP9 $a$ in the resistance to pyrethroids. a Differential expression by quantitative reversetranscription polymerase chain reaction of $C Y P 6 P 9 a$ in the hybrid strain after exposition to permethrin and deltamethrin compared with the susceptible strain FANG; An asterisk indicates significant high differential expression of $C Y P 6 P 9 a$ gene in comparison with
(B)

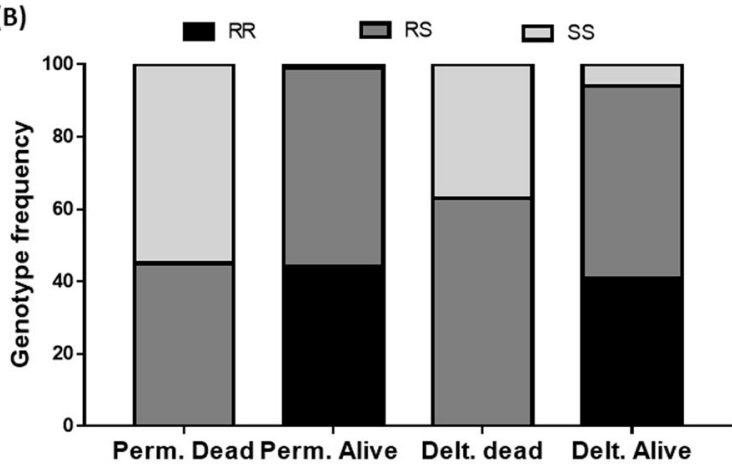

susceptible strain, NS not significant. b Distribution of the CYP6P9a genotypes according to resistance phenotypes. FANG/FUMOZ-R represents a line obtained from the crossing between female FANG and male FUMOZ-R whereas FUMOZ-R/FANG represents a line obtained from the crossing between female FUMOZ-R and male FANG; Perm permethrin, Delt deltamethrin. 
(A)

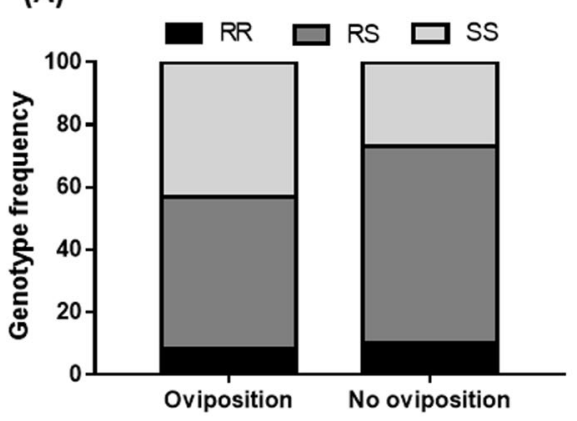

(D)

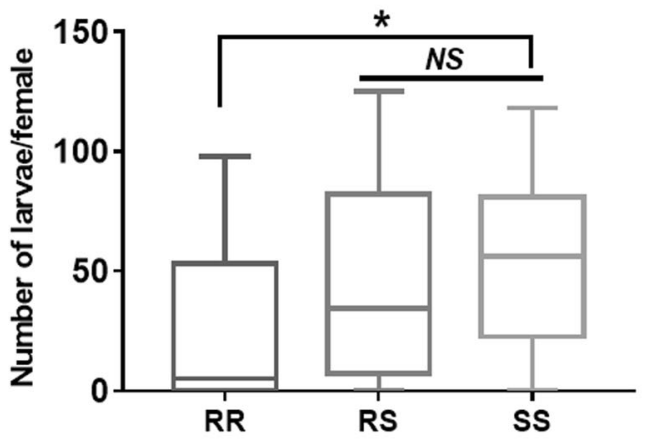

Fig. 2 Fecundity and fertility of females with different genotypes of the CYP6P9 $a$ gene. a, b Schematic representation of the impact of CYP6P9a genotypes on egg-laying success with odd ratio (OR). c Number of eggs laid by the CYP6P9a-RR, CYP6P9a-RS and CYP6P9a-SS genotypes. d Number of larvae generated by females from each genotype. e Comparison of the mean number of eggs laid and hatching rate between the three genotypes. Median value with

Table 1 Assessment of the association between $C Y P 6 P 9 a$ genotypes and the ability of females to lay eggs.

\begin{tabular}{lrl}
\hline Combination of genotypes at the & \multicolumn{2}{c}{ Level of association } \\
\cline { 2 - 3 } CYP6P9 $a$ locus & \multicolumn{1}{c}{ Odds ratio } & $p$ value \\
\hline SS vs. RR & $2.0(0.7-5.7)$ & 0.15 \\
SS vs. RS & $2.04(1.1-3.8)$ & $0.01^{*}$ \\
RS vs. RR & $1(0.4-2.6)$ & 0.57 \\
S vs. R & $1.4(0.8-2.6)$ & 0.1
\end{tabular}

SS homozygote susceptible, $R R$ homozygote resistant, $R S$ heterozygote.

An asterisk indicates significant difference $p<0.05$.

(Table 1 and Fig. 2b) suggesting a non-additional burden of the CYP6P9a_R allele on fecundity.

Furthermore, the median number of eggs laid per female for CYP6P9a_RR was $53.7 \pm 10.05(\min =7$; $\max =124)$. The median was $69.58 \pm 5.04$ (ranged from 4 to 137) for CYP6P9a_RS while CYP6P9a_SS laid $74.52 \pm 4.06$ eggs per female (ranged from 8 to 185) (Fig. 2c). However, a Kruskall-Wallis non-parametric test showed no statistical
(C)

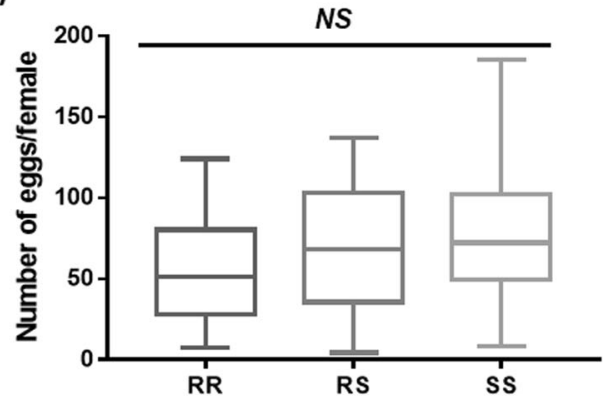

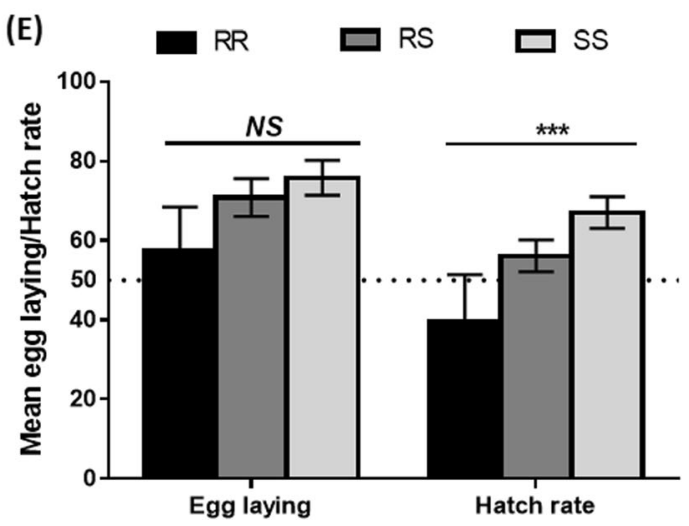

interquartile range is shown for each distribution. Dotted line indicates females for which at least 50 eggs or larvae were obtained. Difference between genotypes was not significant in term of eggs laying by Kruskal-Wallis non-parametric test whereas the number of larvae produced, and the hatch rate differed significantly. ***Significant difference at $p<0.001$; *significant difference at $P<0.05$; NS not significant.

difference $(p=0.2)$ (Fig. 2e). Concerning the viability of eggs laid, RR mosquitoes produced $28.0 \pm 10.31$ larvae $(\min =0 ; \max =98)$ corresponding to a hatch rate of $39 \pm$ $11.86 \%$. For RS mosquitoes the mean number of larvae was $43.7 \pm 4.9$ larvae $(\min =0 ; \max =125)$ corresponding to a hatch rate of $56.1 \pm 4.0 \%$ while SS mosquitoes produced $52.6 \pm 4.0$ larvae $(\min =0 ; \max =118)$ corresponding to a hatch rate of $67.0 \pm 3.9 \%$ (Fig 2d). The mean number of larvae was lower in $\mathrm{RR}(P=0.02)$ compared with other genotypes as well as for the hatch rate $(p<0.04)$ (Fig. 2e).

\section{Level of association between the CYP6P9a_R resistant allele and larval development}

Egg-hatching occurred 2 days post-oviposition and development time from the larvae to the pupae was $12.5 \pm$ 4.5 days overall (Fig. 3). Genotyping of 100 randomly collected larvae for each L1, L2, L3 and L4 stages at $\mathrm{F}_{8}$ generation revealed a significant and consistent decrease of the resistant allele CYP6P9a-R from L1 to L4, indicating greater mortality or slower development of the resistant mosquitoes during this immature stage. A reduction of RR 

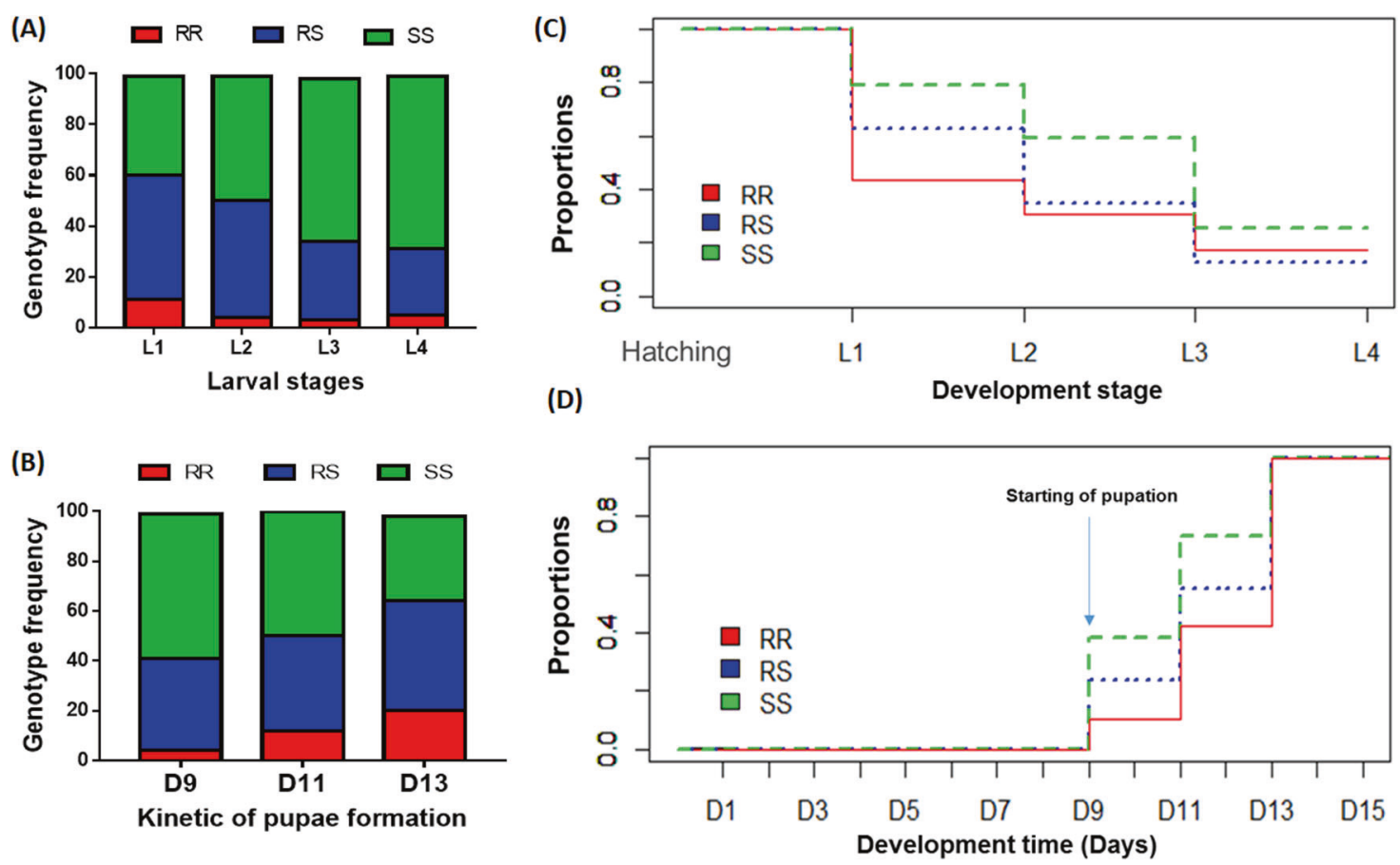

Fig. 3 Distribution of the CYP6P9 $a$ genotypes at different timepoints of the development of immature stages. a Stacked bar plot of the variation in genotypes frequency during the development of larvae (L1, L2, L3, and L4 represent different larval stages) and pupae formation (b); $\mathbf{c}$ the proportion of larvae surviving at each developmental

stage from hatching (day 1) to formation of the pupae; $\mathbf{d}$ the proportion of pupae obtained in day 9, day 11 and day 13 of development. Coloured bars and lines indicate respectively CYP6P9a-RR, CYP6P9a-RS and CYP6P9a-SS genotypes.

Fig. 4 Influence of $C Y P 6 P 9 a$ on the adult longevity of $A n$. funestus. Distribution of CYP6P9a genotypes (a) and alleles (b) at different time in the survived mosquitoes; dotted line indicates a frequency of $50 \%$ for the resistant and susceptible alleles. c Differential expression by quantitative reversetranscription polymerase chain reaction of CYP6P9a genes in alive mosquitoes at different time-points compared with the susceptible lab strain FANG. Error bars represent standard error of the mean; An asterisk indicates statistically significant differential expression of the gene in comparison with susceptible strain; NS not significant.
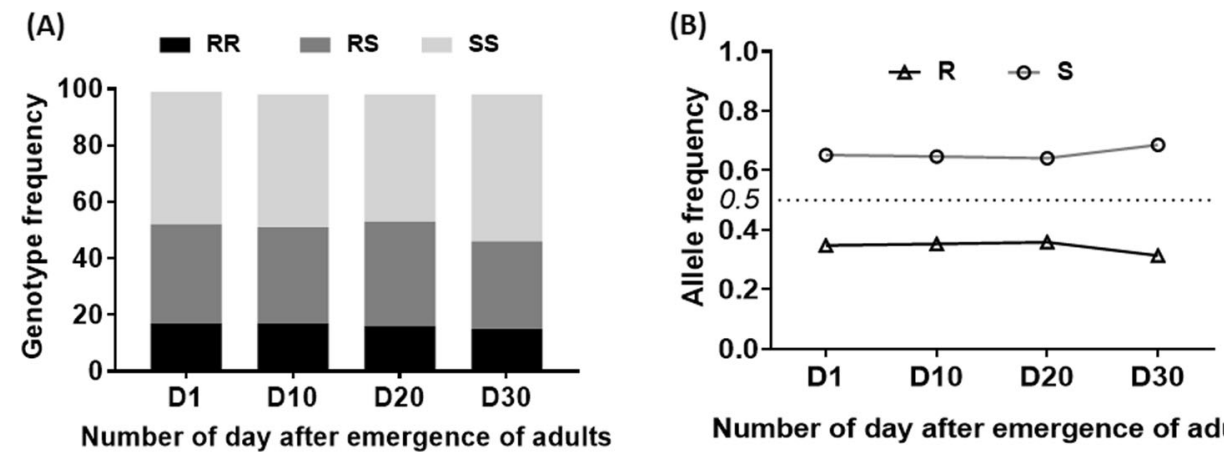

Number of day after emergence of adults

(c)

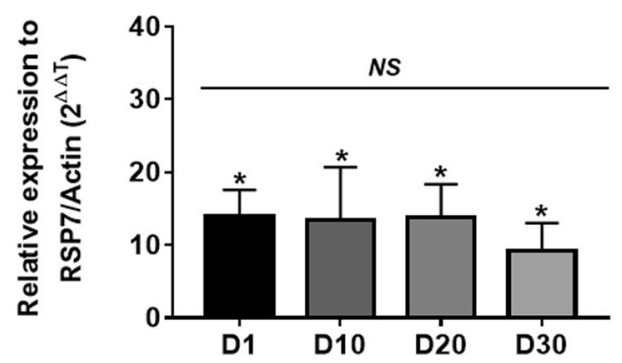

Number of days after emergence of adults

was observed from L1 (11\%) to L4 (5\%) although this was not significant, possibly due to the low number of $\operatorname{RR}\left(\chi^{2}=\right.$ 1.7; $P=0.2$ ) (Fig. 4a, b). A statistically significant decrease of the RS genotype was consistently observed from L1 $(49 \%)$ to $\mathrm{L} 4(27 \%)\left(\chi^{2}=7.2 ; P=0.007\right)$ together with a significant increase of the homozygote susceptible genotype 
Table 2 Association between CYP6P9a genotypes/alleles and pupae formation.

\begin{tabular}{|c|c|c|c|c|c|c|}
\hline \multirow{2}{*}{$\begin{array}{l}\text { Combination of genotypes at } \\
\text { the } C Y P 6 P 9 a \text { locus }\end{array}$} & \multicolumn{2}{|l|}{ Day 9, Day 11} & \multicolumn{2}{|l|}{ Day 9, Day 13} & \multicolumn{2}{|c|}{ Day 11, Day 13} \\
\hline & Odds ratio & $p$ value & Odds ratio & $p$ value & Odds ratio & $p$ value \\
\hline SS vs. RR & $3.0(0.5-16.3)$ & 0.2 & $7.5(1.5-34.4)$ & $0.007 *$ & $2.5(0.6-1.9)$ & 0.1 \\
\hline SS vs. RS & $1.2(0.9-2.2)$ & 0.08 & $2.03(0.9-4.9)$ & 0.08 & $1.7(0.7-3.9)$ & 0.2 \\
\hline RS vs. RR & $2.5(0.5-14.3)$ & 0.3 & $3.7(0.7-18.7)$ & 0.09 & $1.4(0.5-4.5)$ & 0.5 \\
\hline $\mathrm{S}$ vs. $\mathrm{R}$ & $1.5(0.8-2.8)$ & 0.1 & $2.5(1.4-4.6)$ & $0.002 *$ & $1.7(0.9-3.0)$ & $0.05^{*}$ \\
\hline
\end{tabular}

An asterisk indicates significant difference.

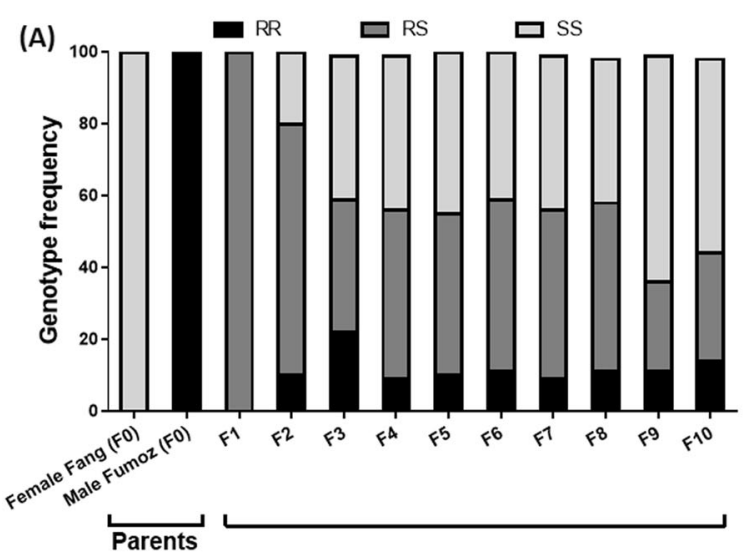

Fig. 5 Evaluation of the reversal to susceptibility in the hybrid colony FANG/FUMOZ-R. Changes in the CYP6P9a genotypes (a) and allele (b) for ten generations in the insecticides free environment.

SS from L1 $(40 \%)$ to L4 (68\%) $\left(\chi^{2}=12.15 ; P=0.0004\right)$ supporting a significant fitness cost of $C Y P 6 P 9 a$ on the larval development of resistant mosquitoes.

Pupae were obtained from 9 days post-hatching (pupae day 9) to 17 days (pupae day 17) with most pupation (more than $75 \%$ ) observed at 11 days post-hatching (pupae day 11). Assessment of the rate of pupae formation by comparing the frequency of the CYP6P9a genotypes in the pupae obtained in day 9, day 11 and day 13 showed a consistent decrease of the homozygote susceptible SS genotype from day $9(58 \%)$ to day $11(34 \%)\left(\chi^{2}=1.73\right.$; $P=0.19)$ together with a significant increase of the RR genotype and RS from day 9 to day $13\left(\chi^{2}=11.17 ; P=\right.$ 0.0008 ) confirming that homozygote susceptible mosquitoes developed significantly faster than RR and RS mosquitoes (Fig. 4c, d). Assessment of the OR for pupae formation further supported that CYP6P9a-SS mosquitoes developed significantly faster than CYP6P9a-RR (OR = $2.50 ; p<0.01)$ whereas there was no difference with CYP6P9a-RS $(\mathrm{OR}=1.18 ; p<0.6)$ (Table 2).

\section{Assessment of the association between CYP6P9a-R allele and adult longevity}

In average 100 alive mosquitoes were genotyped at day 1 , day 10, day 20 and day 30 after the adult emergence to assess the association between the CYP6P9a-R allele and

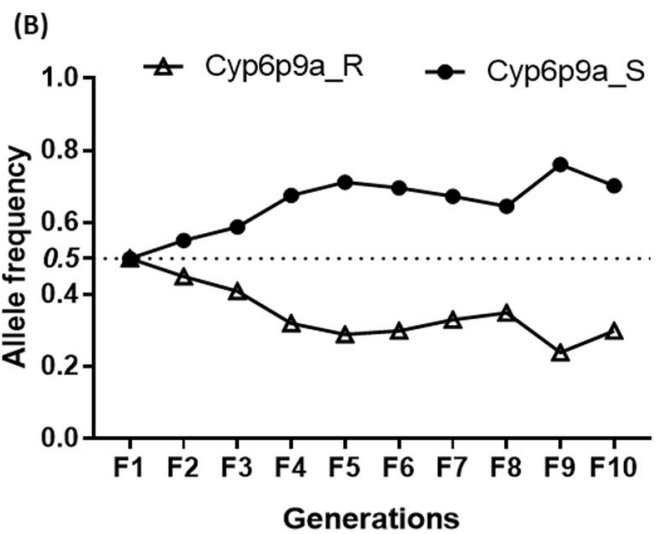

$\mathrm{F}$ represents each generation; dotted line indicates a frequency of $50 \%$ for the resistant and susceptible alleles.

adult longevity. Comparison of genotypes frequency showed no difference in the distribution of genotypes $\left(\chi^{2}=\right.$ $1.6 ; p=0.9$ ) (Fig. 4a) and alleles $\left(\chi^{2}=0.65 ; p=0.88\right.$ ) from day 1 to day 30 (Fig. 4b). In addition, assessment of the OR showed no difference in the life span of SS compared with $\mathrm{RR}(\mathrm{OR}<1.1 ; p>0.4)$ and $\mathrm{RS}(\mathrm{OR}<1.1 ; p>0.2)$ (Table $\mathrm{S} 1$ ). Evaluation of the expression level of CYP6P9a at the same time-points showed no significant difference of the level of expression of this gene in day 1 (fold-change $(\mathrm{FC})=$ $14.03 \pm 3.50)$, day $10(\mathrm{FC}=13.4 \pm 7.2)$, day $20(\mathrm{FC}=$ $13.7 \pm 4.6)$, day $30 \quad(\mathrm{FC}=9.2 \pm 3.8) \quad(F=1.08 ; \quad \mathrm{df}=3$; $p=0.4$ ) suggesting that over-expression of this $\mathrm{P} 450$ gene is not affecting the longevity of female mosquitoes (Fig. 4c).

\section{Assessment of the reversal to susceptibility}

The fitness cost of the CYP6P9a-R was also investigated in cage experiments to assess a potential reversal to susceptibility by examining the changes in the frequency of this allele over ten generations in the absence of insecticide selection pressure. A frequency of $50 \%$ of the resistant allele was confirmed in the $F_{1}$ generation of the FANG/ FUMOZ-R as well as a $50 \%$ for the susceptible allele. A significant and consistent increase in the proportion of homozygote susceptible mosquitoes was observed from $F_{2}(20 \%)$ to $F_{10}(54 \%)\left(\chi^{2}=6.2 ; P=0.01\right)$ (Fig. 5a) suggesting a reversal to susceptibility. This was supported by 
an increase in the frequency of the susceptible allele from $F_{1}(50 \%)$ to $F_{10}(70 \%)\left(\chi^{2}=4.3 ; P=0.03\right)$ (Fig. $5 \mathrm{~b}$ and Table S2).

\section{Discussion}

In this study, using the recently designed diagnostic assay for $C Y P 6 P 9 a$ gene, we investigated the fitness cost associated with $\mathrm{P} 450$-based resistance on various life traits of malaria vectors using laboratory strains of An. funestus revealing a significant cost imposed by $\mathrm{P} 450$-mediated resistance.

\section{Association between the CYP6P9a-based resistance and fecundity/fertility}

The results obtained in this study suggest that $C Y P 6 P 9 a$ induces a reduction in mosquitoes' fecundity and fertility. Same observation of reduced fecundity caused by metabolic based resistance was recently reported for the L119FGSTe2 marker (Tchouakui et al. 2018). This reduced performance of RR mosquitoes in laying eggs could be associated to a reduction in the rate of insemination of resistant mosquitoes compared with the susceptible as previously reported (Brito et al. 2013). Several studies have previously reported a reduction in the number of eggs laid by insecticide resistant strains when compared with the susceptible strain (Brewer and Trumble 1991; Bouvier et al. 2001). In this study, homozygotes resistant mosquitoes displayed a significant lower viability of eggs compared with other genotypes as observed in Ae. aegypti (Mebrahtu et al. 1997).

\section{Effect of the CYP6P9a-R resistant allele on the developmental time of the larvae}

The developmental time of the larvae is a primary aspect of fitness in disseminating mosquito populations (Charlesworth 1994) as the survival rate of larvae or pupae might be reduced in the presence of natural predators or parasites (Agnew and Koella 1999). We observed a greater mortality/ slower development of the resistant mosquitoes during larval development compared with the susceptible ones. This is a clear evidence of fitness cost imposed by P450based metabolic resistance in mosquitoes. A range of environmental factors can affect the developmental time and survivorship of larvae and pupae including temperature, nutrition and larval density (Lyimo et al. 1992; Reisen 1995). In our study, temperature was controlled $\left(25 \pm 2{ }^{\circ} \mathrm{C}\right)$, nutrition and larval density as well. In addition, mosquitoes of the three genotypes were maintained together in the same containers thus limiting all the confounding factors from environmental conditions. A greater mortality associated with a slower development of mosquitoes with the CYP6P9a-R resistant allele could be linked to the competition for food. Probably, despite the fact that all the three genotypes were maintained in the same larval bowl, larvae with $C Y P 6 P 9 a-\mathrm{R}$ resistant allele were less competitive for food and space compared with those with the susceptible allele and therefore, developed significantly slower. As observed previously in resistant Culex pipiens for carboxylesterase-mediated metabolic resistance (Foster et al. 2003), the over-expression of $C Y P 6 P 9 a$ is probably linked with a decreased locomotive performance limiting the ability of mosquitoes with the resistant allele to move faster to feed. All this together could explain the longer developmental time observed in CYP6P9a-RR mosquitoes compared with RSs CYP6P9a-RS and CYP6P9a-SS susceptible mosquitoes. A similar high fitness cost was previously reported for target-site resistance such as $k d r$ in the dengue vector Aedes aegypti (Brito et al. 2013) but this study provides the first evidence that cytochrome P450based metabolic resistance induces a significant fitness cost on larval development in malaria vectors. The high fitness cost of the CYP6P9a-R resistance allele on the larval mortality and/or time of development of immature stages of resistant mosquitoes suggests that a resistance management strategy implemented before the allele becomes fixed in the population could effectively reduce $\mathrm{P} 450$-mediated metabolic resistance in the field. In contrast, a recent study on the impact of GST-based metabolic resistance on larval development found a RS advantage in term of developmental time compared with RR and susceptible mosquitoes for the L119F-GSTe2 marker (Tchouakui et al. 2018). This indicates that the cost of metabolic resistance on the physiological traits of mosquitoes can vary from one enzyme family to another and highlights the necessity to avoid extrapolation and to analyse such fitness cost in more metabolic resistance genes.

\section{Association between the CYP6P9a resistance marker and female longevity}

In contrast with other life traits, there was no association between the CYP6P9a-R resistant allele and adult longevity. Although this need to be assessed in field condition, this observation may suggest that the impact of CYP6P9a-R on the vectorial capacity of resistant mosquitoes might be less pronounced than that observed for the L119F-GSTe2 mutation which was shown to increase the longevity of resistant mosquitoes (Tchouakui et al. 2018). Because the vectors have to live sufficiently longer to allow the parasite to develop until the infective stage (McCarroll and Hemingway 2002), longevity of adult vectors is a key life-trait for which a change due to fitness cost could impact the disease 
transmission. Rivero et al. reported that P450 monoxygenases and the GSTs particularly could drastically alter ROS levels in insects, albeit in radically opposite ways (Rivero et al. 2010). GSTs are known to protect mosquitoes against oxidative stress which results in the increase longevity whereas the increased activity of monoxygenases is associated with increase oxidative stress in mosquitoes (de Montellano 2015). The increase oxidase stress due to overproduction of monoxygenases could therefore reduce the longevity of insects although no such impact was seen in this study. Further studies with field populations will help further assess the extent of the effect of CYP $6 P 9 a$ gene on the life span of resistant mosquitoes in natural conditions.

\section{Monitoring the reversal to susceptibility}

Reversion to susceptibility is expected if the resistant gene harbours a fitness cost in an insecticide-free environment. Therefore, once insecticide pressure ceases, the frequency of the resistant allele, and consequently insecticide resistance, will decrease because of the fitness cost of insecticide resistance on mosquito's life traits. Knowledge of the reversal rate for insecticides such as pyrethroids is therefore crucial before implementing any resistance management strategy in the field. In this study, significant decrease in the frequency of the CYP6P9a-R resistant allele was observed after ten generations in the insecticidefree environment, which correspond to around 1 year. As previously observed (Saavedra-Rodriguez et al. 2012), this reduction in the resistant allele frequency could be attributed either to the accumulation of deleterious effects observed in some life traits of the vector as noticed for fecundity and larval development here or to the pleiotropic effect of other genes very close to $C Y P 6 P 9 a$ such as CYP6P9b since this gene is duplicated in An. funestus. Mating, copulation and insemination efficiency are other key factors which were not assessed in this study but which could have contributed to the reversal observed since female anopheles are inseminated only once during their life span. In these latter cases, males must be able to compete for copula, as the first to inseminate the female will increase the chance of propagating its genes. In the mosquito Culex pipiens males from a susceptible strain showed an advantage when competing for mating compared with males bearing three distinct organophosphate resistant genotypes (Berticat et al. 2002). Similarly, $k d r$ and $R d l$ resistant males An. gambiae were shown to exhibit a lower mating competitiveness than susceptible ones (Platt et al. 2015). If such reduced mating competitiveness is also observed for this An. funestus strain, this could have contributed to the reversal noticed here. Such reversal to susceptibility suggests that resistance management strategies such as insecticide rotation could help to reverse
CYP6P9a-mediated metabolic resistance if implemented early. However, reversal rates can vary and may be very slow or impossible, particularly when an insecticide has been used for several years. For example, in Sri Lanka, the extensive use of DDT for malaria control for about 20 years up to the 1960 s selected a resistance in An. culicifacies s.l. and An. subpictus. For this reason, DDT was replaced by malathion in the early 1970s and DDT resistance reverted very slowly towards susceptibility from $80 \%$ resistance in the 1970 s to about $50 \%$ in the 1990s (Corbel and N'Guessan 2013). The same pattern of result was obtained in West Africa where a reversion of the resistance was observed in Northern Nigeria 6 years after the discontinuation of dieldrin spraying in An. gambiae population (Hamon and Garrett-Jones 1963). In Northern Nigeria, after 20-24 month of massive used of dieldrin, RR mosquitoes was the only ones remaining in the An. gambiae population with only few RSs. But, after 6 months in the absence of dieldrin, the homozygote susceptible genotype took over. Similar results were reported for An. culicifacies in India (Bhatia and Deobhankar 1963). However, the same allele at the $r d l$ locus has been reported to be maintained in field populations in Sri Lanka despite the withdrawal of cyclodiene insecticides for mosquito control for more than 30 years (Roush and Mckenzie 1987). Altogether these variations indicate that knowledge of the reversal rate of an insecticide is crucial for implementing any resistance management strategy in the field based on rotation of insecticides.

\section{Conclusion}

This study has investigated the fitness cost of P450-based metabolic resistance to pyrethroids in a major malaria vector revealing significant fitness cost for fecundity, fertility and the larval development of resistant mosquitoes. This fitness cost was further supported by the observation of a return to susceptibility in the absence of insecticide over ten generations (around 1 year) showing that if suitable resistance management strategies such as rotation was implemented, P450-based resistance could be managed. This should encourage future strategies using non-pyrethroid-based LLINs to reduce the selection pressure and allow such rotation to slow the spread of pyrethroid resistance.

\section{Data availability}

Underlying data is available here DOI (https://doi.org/10. 5061/dryad.pnvx0k6j4).

Funding This study was funded by the Wellcome Trust (Welcome senior 101893/Z/13/Z) awarded to CSW. 
Author contributions CSW conceived and designed the study; MaT carried out the sample collection; MaT, DD and WT reared and maintained the strain in the insectary; MaT, MiT, LMJM and MJW performed the molecular analyses; MaT, JMR and CSW analysed the data; MaT and CSW wrote the paper with contributions from FN. All authors read and approved the paper.

\section{Compliance with ethical standards}

Conflict of interest The authors declare that they have no conflict of interest.

Publisher's note Springer Nature remains neutral with regard to jurisdictional claims in published maps and institutional affiliations.

Open Access This article is licensed under a Creative Commons Attribution 4.0 International License, which permits use, sharing, adaptation, distribution and reproduction in any medium or format, as long as you give appropriate credit to the original author(s) and the source, provide a link to the Creative Commons license, and indicate if changes were made. The images or other third party material in this article are included in the article's Creative Commons license, unless indicated otherwise in a credit line to the material. If material is not included in the article's Creative Commons license and your intended use is not permitted by statutory regulation or exceeds the permitted use, you will need to obtain permission directly from the copyright holder. To view a copy of this license, visit http://creativecommons. org/licenses/by/4.0/.

\section{References}

Agnew P, Koella JC (1999) Life history interactions with environmental conditions in a host-parasite relationship and the parasite's mode of transmission. Evol Ecol 13(1):67-91

Alout H, Dabire RK, Djogbenou LS, Abate L, Corbel V, Chandre F et al. (2016) Interactive cost of Plasmodium infection and insecticide resistance in the malaria vector Anopheles gambiae. Sci Rep 6:29755

Alout H, Yameogo B, Djogbenou LS, Chandre F, Dabire RK, Corbel V et al. (2014) Interplay between Plasmodium infection and resistance to insecticides in vector mosquitoes. J Infect Dis 210:1464-1470

Amenya DA, Naguran R, Lo TC, Ranson H, Spillings BL, Wood OR et al. (2008) Over expression of a cytochrome P450 (CYP6P9) in a major African malaria vector, Anopheles Funestus, resistant to pyrethroids. Insect Mol Biol 17:19-25

Amin A, White G (1984) Relative fitness of organophosphate-resistant and susceptible strains of Culex quinquefasciatus Say (Diptera: Culicidae). Bull Entomol Res 74:591-598

Argentine J, Clark JM, Ferro D (1989) Relative fitness of insecticideresistant Colorado potato beetle strains (Coleoptera: Chrysomelidae). Environ Entomol 18:705-710

Assogba BS, Djogbenou LS, Milesi P, Berthomieu A, Perez J, Ayala D et al. (2015) An ace-1 gene duplication resorbs the fitness cost associated with resistance in Anopheles gambiae, the main malaria mosquito. Sci Rep 5:14529

Berticat C, Boquien G, Raymond M, Chevillon C (2002) Insecticide resistance genes induce a mating competition cost in Culex pipiens mosquitoes. Genet Res 79:41-47

Bhatia S, Deobhankar R (1963) Reversion of dieldrin-resistance in the field population of A. culicifacies in Maharashtra State (erstwhile Bombay State), India. Indian J Malariol 17:339-351

Bhatt S, Weiss D, Cameron E, Bisanzio D, Mappin B, Dalrymple U et al. (2015) The effect of malaria control on Plasmodium falciparum in Africa between 2000 and 2015. Nature 526:207-211
Bouvier JC, Bues R, Boivin T, Boudinhon L, Beslay D, Sauphanor B (2001) Deltamethrin resistance in the codling moth (Lepidoptera: Tortricidae): inheritance and number of genes involved. Heredity $87: 456-462$

Brewer MJ, Trumble JT (1991) Inheritance and fitness consequences of resistance to fenvalerate in Spodoptera exigua (Lepidoptera: Noctuidae). J Econ Entomol 84(6):1638-1644.

Brito LP, Linss JG, Lima-Camara TN, Belinato TA, Peixoto AA, Lima JB et al. (2013) Assessing the effects of Aedes aegypti kdr mutations on pyrethroid resistance and its fitness cost. PLoS ONE 8:e60878

Charlesworth B (1994) Evolution in age-structured populations. Cambridge University Press, Cambridge

Corbel V, N'Guessan R (2013) Distribution, mechanisms, impact and management of insecticide resistance in malaria vectors: a pragmatic review. In: Anopheles mosquitoes-new insights into malaria vectors. IntechOpen

de Montellano, PRO (2015) Substrate oxidation by cytochrome P450 enzymes. In Cytochrome P450. Springer, Cham, pp. 111-176

Edi CV, Djogbenou L, Jenkins AM, Regna K, Muskavitch MA, Poupardin R et al. (2014) CYP6 P450 enzymes and ACE-1 duplication produce extreme and multiple insecticide resistance in the malaria mosquito Anopheles gambiae. PLoS Genet 10:e1004236

Foster SP, Young S, Williamson MS, Duce I, Denholm I, Devine GJ (2003) Analogous pleiotropic effects of insecticide resistance genotypes in peach-potato aphids and houseflies. Heredity 91:98-106

Hamon J, Garrett-Jones C (1963) Resistance to insecticides in the major malaria vectors and its operational importance. Bull World Health Organ 28:1

Hemingway J (2014) The role of vector control in stopping the transmission of malaria: threats and opportunities. Philos Trans $\mathrm{R}$ Soc Lond B Biol Sci 369:20130431

Hunt R, Brooke B, Pillay C, Koekemoer L, Coetzee M (2005) Laboratory selection for and characteristics of pyrethroid resistance in the malaria vector Anopheles funestus. Med Vet Entomol 19:271-275

Ibrahim SS, Riveron JM, Bibby J, Irving H, Yunta C, Paine MJ et al. (2015) Allelic variation of cytochrome P450s drives resistance to bednet insecticides in a major malaria vector. PLoS Genet 11: e1005618

Irving H, Wondji CS (2017) Investigating knockdown resistance (kdr) mechanism against pyrethroids/DDT in the malaria vector Anopheles funestus across Africa. BMC Genet 18:76

Kwiatkowska RM, Platt N, Poupardin R, Irving H, Dabire RK, Mitchell S et al. (2013) Dissecting the mechanisms responsible for the multiple insecticide resistance phenotype in Anopheles gambiae s.s., M form, from Vallée du Kou, Burkina Faso. Gene 519:98-106

Livak KJ (1984) Organization and mapping of a sequence on the Drosophila melanogaster $\mathrm{X}$ and $\mathrm{Y}$ chromosomes that is transcribed during spermatogenesis. Genetics 107:611-634

Lyimo E, Takken W, Koella J (1992) Effect of rearing temperature and larval density on larval survival, age at pupation and adult size of Anopheles gambiae. Entomologia experimentalis et applicata 63:265-271

Martinez-Torres D, Chandre F, Williamson MS, Darriet F, Berge JB, Devonshire AL et al. (1998) Molecular characterization of pyrethroid knockdown resistance $(\mathrm{kdr})$ in the major malaria vector Anopheles gambiae s.s. Insect Mol Biol 7:179-184

Martins AJ, Ribeiro CD, Bellinato DF, Peixoto AA, Valle D, Lima JB (2012) Effect of insecticide resistance on development, longevity and reproduction of field or laboratory selected Aedes aegypti populations. PLoS ONE 7:e31889

McCarroll L, Hemingway J (2002) Can insecticide resistance status affect parasite transmission in mosquitoes? Insect Biochem Mol Biol 32:1345-1351 
Mebrahtu YB, Norem J, Taylor M (1997) Inheritance of larval resistance to permethrin in Aedes aegypti and association with sex ratio distortion and life history variation. Am J Trop Med Hyg $56: 456-465$

Mitchell SN, Stevenson BJ, Muller P, Wilding CS, Egyir-Yawson A, Field SG et al. (2012) Identification and validation of a gene causing cross-resistance between insecticide classes in Anopheles gambiae from Ghana. Proc Natl Acad Sci USA 109:6147-6152

Morgan JC, Irving H, Okedi LM, Steven A, Wondji CS (2010) Pyrethroid resistance in an Anopheles funestus population from Uganda. PLoS ONE 5:e11872

Okoye PN, Brooke BD, Koekemoer LL, Hunt RH, Coetzee M (2008) Characterisation of DDT, pyrethroid and carbamate resistance in Anopheles funestus from Obuasi, Ghana. Trans R Soc Trop Med Hyg 102:591-598

Platt N, Kwiatkowska RM, Irving H, Diabaté A, Dabire R, Wondji CS (2015) Target-site resistance mutations (kdr and RDL), but not metabolic resistance, negatively impact male mating competiveness in the malaria vector Anopheles gambiae. Heredity 115:243

Ranson H, Jensen B, Vulule JM, Wang X, Hemingway J, Collins FH (2000) Identification of a point mutation in the voltage-gated sodium channel gene of Kenyan Anopheles gambiae associated with resistance to DDT and pyrethroids. Insect Mol Biol 9:491-497

Ranson H, N'guessan R, Lines J, Moiroux N, Nkuni Z, Corbel V (2011) Pyrethroid resistance in African anopheline mosquitoes: what are the implications for malaria control? Trends Parasitol 27:91-98

Reisen WK (1995) Effect of temperature on Culex tarsalis (Diptera: Culicidae) from the Coachella and San Joaquin valleys of California. J Med Entomol 32:636-645

Rivero A, Vezilier J, weill M, Read AF, Gandon S (2010) Insecticide control of vector-borne diseases: when is insecticide resistance a problem? PLoS Pathog 6:e1001000

Riveron JM, Yunta C, Ibrahim SS, Djouaka R, Irving H, Menze BD, et al. (2014) A single mutation in the GSTe2 geneallows tracking of metabolically based insecticide resistance in a major malaria vector. Genome Biol 15:R27

Riveron JM, Irving H, Ndula M, Barnes KG, Ibrahim SS, Paine MJ et al. (2013) Directionally selected cytochrome P450 alleles are driving the spread of pyrethroid resistance in the major malaria vector Anopheles funestus. Proc Natl Acad Sci USA 110:252-257

Riveron JM, Watsenga F, Irving H, Irish SR, Wondji CS (2018) High Plasmodium infection rate and reduced bed net efficacy in multiple insecticide-resistant malaria vectors in Kinshasa, Democratic Republic of Congo. J Infect Dis 217(2):320-328

Roush RT, Mckenzie JA (1987) Ecological genetics of insecticide and acaricide resistance. Annu Rev Entomol 32:361-380

Saavedra-Rodriguez K, Suarez AF, Salas IF, Strode C, Ranson H, Hemingway J et al. (2012) Transcription of detoxification genes after permethrin selection in the mosquito Aedes aegypti. Insect Mol Biol 21:61-77

Schmittgen TD, Livak KJ (2008) Analyzing real-time PCR data by the comparative C(T) method. Nat Protoc 3:1101-1108

Tchouakui M, Chiang M-C, Ndo C, Kuicheu CK, Amvongo-Adjia N, Wondji MJ et al. (2019) A marker of glutathione S-transferasemediated resistance to insecticides is associated with higher Plasmodium infection in the African malaria vector Anopheles funestus. Sci Rep 9:5772

Tchouakui M, Riveron JM, Djonabaye D, Tchapga W, Irving H, Takam PS et al. (2018) Fitness costs of the glutathione Stransferase epsilon 2 (L119F-GSTe2) mediated metabolic resistance to insecticides in the major African malaria vector Anopheles funestus. Genes 9:645

Weedall GD, Mugenzi LM, Menze BD, Tchouakui M, Ibrahim SS, Amvongo-Adjia N et al. (2019) A cytochrome P450 allele confers pyrethroid resistance on a major African malaria vector, reducing insecticide-treated bednet efficacy. Sci Transl Med 11:eaat7386

WHO (1998) Test procedures for insecticide resistance monitoring in malaria vectors, bio-efficacy and persistence of insecticides on treated surfaces: report of the WHO informal consultation, WHO, Geneva

WHO (2013) Test procedures for insecticide resistance monitoring in malaria vector mosquitoes. World Health Organization, Rep Ser

WHO (2018) World malaria report 2018

Wondji CS, Dabire RK, Tukur Z, Irving H, Djouaka R, Morgan JC (2011) Identification and distribution of a GABA receptor mutation conferring dieldrin resistance in the malaria vector Anopheles funestus in Africa. Insect Biochem Mol Biol 41:484-491 